\title{
A new approach for computing the geometry of the moduli spaces for a Calabi-Yau manifold
}

\author{
Konstantin Aleshkin ${ }^{1,2}$, Alexander Belavin ${ }^{1,3,4}$ \\ ${ }^{1}$ L.D. Landau Institute for Theoretical Physics \\ Akademika Semenova av. 1-A \\ Chernogolovka, 142432 Moscow region, Russia \\ ${ }^{2}$ International School of Advanced Studies (SISSA), via Bonomea 265, 34136 Trieste, Italy \\ ${ }^{3}$ Department of Quantum Physics \\ Institute for Information Transmission Problems \\ Bolshoy Karetny per. 19, 127994 Moscow, Russia \\ ${ }^{4}$ Moscow Institute of Physics and Technology \\ Dolgoprudnyi, 141700 Moscow region, Russia \\ E-mail: kaleshkin@sissa.it, belavin@itp.ac.ru
}

ABstract: It is known that moduli spaces of Calabi-Yau (CY) manifolds are special Kähler manifolds. This structure determines the corresponding low-energy effective theory which arises in superstring compactifications on CY manifolds. In the case, where CY manifold is given as a hypersurface in the weighted projective space, we propose a new procedure for computing the Kähler potential of the moduli space. Our method is based on the fact that the moduli space of CY manifolds is a marginal subspace of the Frobenius manifold which arises on the deformation space of the corresponding Landau-Ginzburg superpotential. 


\section{Contents}

1 Introduction 1

2 Special geometry 3

3 Hypersurface in weighted projective space 5

4 Frobenius manifold structure on the deformation space of $W_{0} \quad 8$

5 Computing the Kähler potential. $\quad 11$

6 Examples $\quad 12$

$\begin{array}{lll}7 & \text { Conclusion } & 17\end{array}$

8 Acknowledgements $\quad 18$

\section{Introduction}

To compute the low-energy Lagrangian of the string theory compactified on a CY manifold, one needs to know the geometry of the corresponding CY moduli space. One way to compute this structure was proposed in [1,2].

As shown in [3], see also [4, 5], the Kähler potential of the metric on the moduli space of CY manifold $X$ is expressed bilinearly through periods of a holomorphic 3-form $\Omega$.

$$
\omega_{\mu}:=\oint_{q_{\mu}} \Omega
$$

where $q_{\mu}$ form a basis of $H_{3}(X, \mathbb{Z})$.

The Kähler potential is computed in two steps.

The first step is to compute periods $\omega_{\mu}$ in a special basis of cycles $q_{\mu}$. It was successfully done for a large number of CY manifolds and Landau-Ginzburg (LG) orbifolds in [2]. The second step is to compute a transition matrix from $\omega_{\mu}$ to the symplectic basis of periods $\Pi_{\mu}$. Then Kähler potential is given by the bilinear form of periods $\Pi_{\mu}$. This second step appears to be highly non-trivial. It was done for the case of the quintic CY manifold in the beautiful paper [1].

In this work, we present an alternative approach to the computation of the Kähler potential for the case where CY manifold is given by a hypersurface $W(x)=0$ in a weighted projective space. More specifically, our results immediately apply to the case when a non-degenerate CY manifold can be defined by a hypersurface $W_{0}(x)=0$ such that the function $W_{0}(x)$ defines an invertible singularity [6], that is $W_{0}(x)$ is a polynomial 
with a minimal possible number (which is 5 for a 3 -fold) of monomials with some further technical restriction, which is discussed in the section 6 .

This approach is based on the connection of the CY manifold with a Frobenius manifold (FM) which arises on the monodromy invariant deformations of the singularity defined by the LG superpotential $W_{0}(x)$ (see, e.g., [7-9]). Marginal elements of the Milnor ring (quasihomogeneous deformations of the singularity) are in one-to-one correspondence with complex structure deformations of the CY manifold $X=\{W(X)=0\} \subset \mathbb{P}^{5}$ and thus with $H^{2,1}(X)$. Monodromy invariant Milnor ring is generated by these elements and is isomorphic to $H^{3}(X)$ where the degree of the Milnor ring element corresponds to the Hodge decomposition. The moduli space of the CY manifold is a marginal subspace of this Frobenius manifold. This fact allows to compute easily the intersection matrix required to compute the Kähler potential.

We also make use of two different basises of the periods $\omega_{\mu}, \sigma_{\mu}$ of the holomorphic volume form $\Omega$ on the CY. The basis $\omega_{\mu}$ is the same as in the papers $[1,2]$. The $\sigma_{\mu}$, in turn, is given by the connection with the chiral ring of the LG theory for the superpotential $W_{0}(x)$.

Namely, the natural basis of the chiral ring gives a basis in the cohomology of the differential $D_{W_{0}}^{ \pm}=\mathrm{d} \pm \mathrm{d} W_{0} \wedge$. Periods $\sigma_{\mu}$ are then chosen as integrals over the dual basis of homology cycles $\Gamma_{\mu}^{ \pm}$, which belong to the relative homology $H_{5}\left(\mathbb{C}, \operatorname{Re} W_{0}(x)= \pm \infty\right)$.

The expression for the Kähler potential is then given in terms of the periods $\sigma_{\mu}$, the holomorphic metric on the Frobenius Manifold and transition matrix $T$ from periods $\omega_{\mu}$ to $\sigma_{\mu}$ (see section 5):

$$
e^{-K}=\sigma_{\mu}^{+} \eta^{\mu \rho} M_{\rho}^{\nu} \overline{\sigma_{\nu}^{-}}, \quad M=T^{-1} \bar{T} .
$$

The basis $\omega_{\mu}$ of periods has the advantage of being taken over homology cycles $q_{\mu}$ with integer coefficients. Therefore, both the Kähler potential and the (holomorphic) metric of the FM are expressed in terms of the same intersection matrix of the corresponding cycles.

$$
\begin{gathered}
e^{-K(\phi)}=\omega_{\mu}(\phi) C^{\mu \nu} \overline{\omega_{\nu}(\phi)}, \\
h_{\alpha \beta}=\omega_{\alpha \mu}(0) C^{\mu \nu} \omega_{\beta \nu}(0),
\end{gathered}
$$

where $\left(C^{-1}\right)_{\mu \nu}=q_{\mu} \cap q_{\nu}$ and $\omega_{\alpha \mu}(\phi)$ are the different periods defined by integration over the same cycles. However, it turns out that $\omega_{\alpha \mu}$ are inconvenient to use in the second equation.

On the contrary, periods of the basis $\sigma_{\mu}$ and the additional periods $\sigma_{\alpha \mu}^{ \pm}$defined as integrals over homology cycles $\Gamma_{\mu}^{ \pm}$with complex coefficients have more sophisticated connection with the Kähler potential, but their relation with the holomorphic metric on the FM is simple.

We demonstrate our method on the famous Quintic hypersurface, Fermat surfaces and CY manifolds which are defined through the invertible singularities.

This paper is structured as follows. In section 2, we recall the notion of the Special Kähler geometry structure arising on the moduli spaces of CY manifolds. In section 3, 
we focus on the case when CY manifold is a hypersurface in a weighted projective space and discuss periods. In section 4 we review the Frobenius manifold structure connected with such CY, and we also introduce the second set of periods. In section 5, we present our main result, the formula for the Kähler potential on the moduli space. In section 6 , we demonstrate the efficiency of our method with some examples.

\section{Special geometry}

Here, we recall the basic facts about special Kähler geometry and how it arises on the CY moduli space. Most of the material can be found in [3-5, 10, 11].

It was shown in [5] that the moduli space $\mathcal{M}$ of complex (or Kähler) structures of a given CY manifold is a special Kähler manifold. That is, if $\mathcal{M}$ is $n$-dimensional, then there are so-called special (projective) coordinates $z^{1} \cdots z^{n+1}$ and a holomorphic homogeneous function $F(z)$ of degree 2 in $z$ called a prepotential such that the Kähler potential $K(z)$ of the moduli space metric is given by

$$
e^{-K(z)}=z^{a} \cdot \frac{\partial \bar{F}}{\partial \bar{z}^{\bar{a}}}-\bar{z}^{\bar{a}} \cdot \frac{\partial F}{\partial z^{a}} .
$$

This metric on the moduli space of complex structures is a metric that naturally arises from B. deWitt metric on a space of metrics on CY manifolds.

\section{Special geometry on the complex structure moduli space}

Consider a CY three-fold $X$. The CY moduli space of $X$ is the space of metric perturbations of $X$ that preserve Ricci-flatness.

Then, the metric on the complex structure CY moduli space can be obtained[5] from natural deWitt metric for pure holomorhic CY metric deformations $\delta g_{\mu \nu}, \delta g_{\bar{\mu} \bar{\nu}}$ and given by

$$
G_{a \bar{b}}=\int_{X} \mathrm{~d}^{6} y g^{1 / 2} g^{\mu \bar{\sigma}} g^{\nu \bar{\rho}} \delta_{a} g_{\mu \nu} \delta_{\bar{b}} g_{\bar{\sigma} \bar{\rho}}
$$

The CY metric deformation with two indices of the same holomorphicity that leave the metric Ricci flat corresponds to elements in $H_{\bar{\partial}}^{0,1}(T X) \simeq H^{2,1}(X)$ :

$$
\delta_{a} g_{\bar{\nu} \bar{\sigma}} \rightarrow \delta_{a} g_{\bar{\nu} \bar{\sigma}} g^{\bar{\sigma} \mu} \mathrm{d} \bar{y}^{\bar{\nu}}=h_{a, \bar{\nu}}^{\mu} \mathrm{d} \bar{y}^{\bar{\nu}} \sim \Omega_{\mu \nu \sigma} h_{a, \bar{\rho}}^{\sigma} \mathrm{d} y^{\mu} \wedge \mathrm{d} y^{\nu} \wedge \mathrm{d} y^{\bar{\rho}} \sim \chi_{a, \mu \nu \bar{\rho}}
$$

We can then rewrite the above metric as

$$
G_{a \bar{b}}=\frac{\int_{X} \chi_{a} \wedge \bar{\chi}_{\bar{b}}}{\int_{X} \Omega \wedge \bar{\Omega}} .
$$

Here indices $a, \bar{b}$ are indices in the deformation space, that is in $H^{2,1}(X)$. Using the following lemma (attributed to Kodaira):

$$
\partial_{a} \Omega=k_{a} \Omega+\chi_{a},
$$


we easily verify that this metric is a Kähler :

$$
G_{a \bar{b}}=-\partial_{a} \partial_{\bar{b}} \ln \int_{X} \Omega \wedge \bar{\Omega}
$$

To obtain[5] the formulae written above, we choose Poincare dual symplectic basises $\alpha_{a}, \beta^{b} \in H^{3}(X, \mathbb{Z})$ and $A^{a}, B_{b} \in H_{3}(X, \mathbb{Z})$ :

$$
\begin{gathered}
\int_{A^{a}} \alpha_{b}=\delta_{b}^{a}, \quad \int_{A^{a}} \beta^{b}=0, \quad \int_{B_{a}} \alpha_{b}=0, \quad \int_{B_{a}} \beta^{b}=\delta_{a}^{b}, \\
A^{a} \cap B_{b}=\delta_{b}^{a}, \quad A^{a} \cap A^{b}=0, \quad B_{a} \cap B_{b}=0 . \\
\int_{X} \alpha_{a} \wedge \beta^{b}=\delta_{a}^{b}, \quad \int_{X} \alpha_{a} \wedge \alpha_{b}=0 \quad \int_{X} \beta^{a} \wedge \beta^{b}=0 .
\end{gathered}
$$

With this, we decompose $\Omega$ as

$$
\Omega=z^{a} \alpha_{a}+F_{b} \beta^{b}
$$

here $z^{a}, F_{b}$ are periods of $\Omega$ :

$$
z^{a}=\int_{A^{a}} \Omega, F_{b}=\int_{B_{b}} \Omega
$$

Substituting this in (2.8) we obtain

$$
e^{-K}=\int_{X} \Omega \wedge \bar{\Omega}=z^{a} \cdot \bar{F}_{\bar{a}}-\bar{z}^{\bar{a}} \cdot F_{a} .
$$

From the same lemma we also obtain

$$
0=\int_{X} \Omega \wedge \partial_{a} \Omega=F_{a}-z^{b} \partial_{a} F_{b} \Longrightarrow F_{a}(z)=\frac{1}{2} \partial_{a}(F(z)),
$$

where $F(z)=1 / 2 z^{b} F_{b}(z)$. Therefore, according to the defnition (2.1) metric $G_{a \bar{b}}=$ $\partial_{a} \bar{\partial}_{\bar{b}} K(z)$ is a special Kähler metric with prepotential $F(z)$ and with special coordinates given by the period vector.

Using the notation for the vector of periods

$$
\Pi=\left(F_{\alpha}, z^{b}\right)
$$

we write the expression for the Kähler potential as

$$
e^{-K(z)}=\Pi_{a} \Sigma^{a b} \overline{\Pi_{b}}
$$

where $\Sigma$ is a symplectic unit, which is an inverse intersection matrix for cycles $A^{a}$ and $B_{b}$.

Also Yukawa couplings can be expressed through the prepotential $F(z)[5]$ as:

$$
\kappa_{a b c}=\partial_{a} \partial_{b} \partial_{c} F(z)=\int_{X} \Omega \wedge \partial_{a} \partial_{b} \partial_{c} \Omega
$$




\section{Kähler potential in an arbitrary basis of periods.}

Using formula (2.13), we can rewrite this expression in any basis of periods

$$
\omega_{\mu}=\int_{q_{\mu}} \Omega
$$

where $q_{\mu} \in H_{3}(X, \mathbb{Z})$, and then

$$
e^{-K}=\omega_{\mu} C^{\mu \nu} \overline{\omega_{\nu}}
$$

where $C^{\mu \nu}$ is the inverse marix of the intersection of cycles.

Thus, to find the Kähler potential, we must compute the periods over a basis of cycles on CY manifold together with their intersection matrix.

\section{Hypersurface in weighted projective space}

Further, we concentrate on the case where the CY manifold is realized as a zero locus of a single polynomial equation in a weighted projective space. In this case, we establish the crucial relationship with the FM structure. We consider CY 3-folds, but most of our result are generalizable to an arbitrary dimension.

Fundamental basis of periods Let $x_{1}, \ldots, x_{5}$ be homogeneous coordinates in a weighted projective space and

$$
X=\left\{x_{1}, \ldots, x_{5} \in \mathbb{P}_{\left(k_{1}, \ldots, k_{5}\right)}^{4} \mid W_{0}(x)=0\right\} .
$$

For some quasi-homogeneous polynomial $W_{0}(x)$,

$$
W_{0}\left(\lambda^{k_{i}} x_{i}\right)=\lambda^{d} W_{0}\left(x_{i}\right),
$$

and

$$
\operatorname{deg} W_{0}(x)=d=\sum_{i=1}^{5} k_{i} .
$$

The last relation ensures that $X$ is a CY manifold. Here $W_{0}(x)$ is the superpotential for the corresponding Landau-Ginzburg (LG) model, and $W_{0}(x)$ defines an isolated singularity in the origin iff the CY manifold is quasi-smooth ${ }^{1}$. The moduli space of complex structures is then given by homogeneous polynomial deformations of this singularity:

$$
W(x, \phi)=W_{0}(x)+\sum_{s=0}^{\mu} \phi^{s} e_{s}(x),
$$

\footnotetext{
${ }^{1}$ Quasi-smoothness means that the only possible singularities of the CY are given by those of the underlying weighted projective space
} 
where $e_{s}(x)$ are monomials of $x$ which have the same weight as $W_{0}(x)$. In this case, the holomorphic 3 -form $\Omega$ is given as a residue of a 5 -form in the underlying affine space $\mathbb{C}^{5}$ :

$$
\begin{aligned}
\Omega=\frac{x_{5} \mathrm{~d} x_{1} \wedge \mathrm{d} x_{2} \wedge \mathrm{d} x_{3}}{\partial W(x) / \partial x_{4}}=\operatorname{Res}_{W(x)=0} \frac{x_{5} \mathrm{~d} x_{1} \cdots \mathrm{d} x_{4}}{W(x)} & = \\
& =\frac{1}{2 \pi i} \oint_{\left|x_{5}\right|=\delta} \operatorname{Res}_{W(x)=0} \frac{\mathrm{d} x_{1} \cdots \mathrm{d} x_{5}}{W(x)},
\end{aligned}
$$

where the last equality is due to the homogeneity of the integrand. Residue is understood as an integral over a small circle in the direction transversal to the hypersurface $W(x)=0$ at each point.

Using explicit expression (3.5) for $\Omega$, we can compute a basis of periods $\omega_{\mu}(\phi)$ as follows [2]. We take a so-called fundamental cycle $q_{1}$, which is a torus in the large complex structure limit:

$$
W(x, \phi)=W_{0}(x)+\phi_{0} \prod x_{i}+\sum_{s=1}^{\mu} \phi^{s} e_{s}(x), \phi_{0}>>1 .
$$

In this limit, we can define an 5-dimensional torus $Q_{1}=\left|x_{i}\right|=\delta_{i}$ surrounding the hypersurface $W(x)=0$ in $\mathbb{C}^{5}$. It corresponds to an 3 -dimensional torus $q_{1} \subset X$. Then the fundamental period is defined as an integral over this cycle

$$
\omega_{1}(\phi):=\int_{q_{1}} \Omega=\int_{Q_{1}} \frac{\mathrm{d} x^{1} \cdots \mathrm{d} x^{5}}{W(x, \phi)}
$$

and is given by a residue in its large $\phi_{0}$ expansion. It was computed for many cases in the work [2].

More periods $\omega_{\mu}$ may be obtained as analytic continuations of $\omega_{1}$ in $\phi$. This can be conveniently done by continuing $\omega_{1}(\phi)$ in a small $\phi_{0}$ region using Mellin-Barnes integrals and using the symmetry of $W_{0}(x)$ afterward.

Namely, there is a group of phase symmetries $\Pi_{X}$ acting diagonally on $x_{i}$ and this action preserves $W_{0}(x)$. It doesn't preserve the deformed polynomial $W(x)$, but we can extend this action to $\mathcal{A}: G \times\left\{\phi^{s}\right\} \rightarrow\left\{\phi^{s}\right\}$ on the parameter space such that $W(x)$ is invariant:

$$
W\left(g \cdot x, \mathcal{A}(g) \cdot \phi_{0}, \mathcal{A}(g) \cdot \phi^{s}\right)=W(x, \phi) .
$$

The moduli space is then at most a factor of the parameter space $\left\{\phi^{s}\right\} / \mathcal{A}$.

This allows to define a set of other periods by analytic continuation,

$$
\omega_{\mu_{g}}(\phi)=\omega_{1}\left(\mathcal{A}(g) \cdot \phi_{0}, \mathcal{A}(g) \cdot \phi^{s}\right) g \in G_{X}
$$

In many cases this construction gives the whole basis of periods for the manifold $X[2]$.

\section{Periods as oscillatory integrals}

The next important step is to transform the integrals for the periods $\int_{q_{\mu}} \Omega$ to the complex 
oscillatory form. First we have

$$
\omega_{\mu}(\phi):=\int_{q_{\mu}} \Omega=\int_{Q_{\mu}} \frac{\mathrm{d}^{5} x}{W(x)}
$$

where $q_{\mu} \in H_{3}(X), Q_{\mu} \in H_{5}\left(\mathbb{C}^{5} \backslash W(x)=0\right)$, and $Q_{\mu}$ is given by a tubular neighbourhood of $q_{\mu}$. Now we can present them in the form

$$
\int_{Q_{\mu}} \frac{\mathrm{d}^{5} x}{W(x)}=\int_{Q_{\mu}^{ \pm}} e^{\mp W(x)} \mathrm{d}^{5} x
$$

where $Q_{\mu}^{ \pm} \in H_{5}\left(\mathbb{C}^{5}, \operatorname{Re} W_{0}(x)= \pm \infty\right)$.

The map $Q_{\mu} \rightarrow Q_{\mu}^{ \pm}$is given by a contour deformation similarly to how it was done in [12] and in [13]. This deformation can be performed due to the existence of a natural isomorphism ${ }^{2}[14]$

$$
H_{3}(X) \rightarrow H_{5}\left(\mathbb{C}^{5} \backslash W(x)=0\right)=H_{5}\left(\mathbb{C}^{5}, \operatorname{Re} W_{0}(x)= \pm \infty\right)_{w \in d \cdot \mathbb{Z}}
$$

Here

$$
H_{5}\left(\mathbb{C}^{5}, \operatorname{Re} W_{0}(x)= \pm \infty\right)_{w \in d \cdot \mathbb{Z}}
$$

is a subgroup of $H_{5}\left(\mathbb{C}^{5}, \operatorname{Re} W_{0}(x)= \pm \infty\right)$, which we describe in the next paragraph. Using this isomorphism, we rewrite the periods as

$$
\omega_{\mu}=\omega_{\mu}^{ \pm}=\int_{Q_{\mu}^{ \pm}} e^{\mp W(x)} \mathrm{d}^{5} x
$$

and from formula (2.16), we obtain

$$
e^{-K}=\omega_{\mu}^{+} C^{\mu \nu} \overline{\omega_{\nu}^{-}}
$$

where $C^{\mu \nu}=q_{\mu} \cap q_{\nu}=Q_{\mu}^{+} \cap Q_{\nu}^{-}$and $Q_{\mu}^{ \pm} \cap Q_{\nu}^{ \pm}=0$. At the moment we do not know how to prove that intersections of the cycles $q_{\mu} \cap q_{\nu}$ and $Q_{\mu}^{+} \cap Q_{\nu}^{-}$do coincide. However, this conjecture agrees with the Cecotti-Vafa formula in [15] for the Kähler potential. Also, we have verified the conjecture by direct computations in a few examples considered in section 6 and comparing our results with obtained earlier in $[1,16]$.

Taking into account that the basis $\omega_{\mu}$ of periods has been already found for a large class of CY manifolds in [2], we need only to know the matrix $C^{\mu \nu}$ to obtain the Kähler potential for the Moduli space of CY manifolds in these cases.

To solve this problem, we will use the fact that the CY moduli space is the marginal subspace of the Frobenius manifold [7] which arises on the monodromy invariant deformations of the singularity given by the LG superpotential $W_{0}(x)$. We will do this in the next sections.

\footnotetext{
${ }^{2}$ We are thankful to A. Givental and V. Vasiliev for explanation of this point.
} 


\section{Frobenius manifold structure on the deformation space of $W_{0}$}

It was conjectured and partially demonstrated by Gepner in [17] that compactifications of superstring theory on CY manifolds invented in [18] are equivalent to compactifications on $N=2$ superconformal field theories.

In the cases considered now they are LG models with superpotential $W_{0}(x)$. The deep connection of these models with singularity theory was established in [19-21] . The Frobenius manifold structure appears on the space of deformations of this singularity.

The polynomial $W_{0}(x)$ in $\mathbb{C}^{5}$, which defines a quasi-smooth CY hypersurface in some 4-dimensional weighted projective space, is a quasi-homogeneous polynomial:

$$
W_{0}\left(\lambda^{k_{i}} x\right)=\lambda^{d} W_{0}(x)
$$

with an isolated singularity in the origin.

We consider the Milnor ring of this singularity

$$
R_{0}=\frac{\mathbb{C}\left[x_{1}, \ldots, x_{5}\right]}{\partial_{1} W_{0}(x) \cdot \ldots \cdot \partial_{5} W_{0}(x)}
$$

and its subring $R_{0}^{Q}$ of elements of degree divisible by degree $d$ of the singularity ${ }^{3}$. We note that this is precisely a subring invariant under the following action of

$$
Q=\mathbb{Z}_{d}: m \cdot x_{j}=e^{\frac{2 \pi i m k_{j}}{d}} x_{j}
$$

where $m \in Q$. Let $e_{\mu}(x)$ be a (vector space) basis of $R_{0}^{Q}$, consisting of homogeneous monomials of the least possible degree. There is a natural multiplication in this ring induced by multiplication of polynomials, and there is also a metric, turning the space of $e_{\mu}(x)$ into a Frobenius algebra.

The metric and struture constants are given by

$$
\begin{aligned}
\eta_{\mu \nu} & =\operatorname{Res} \frac{e_{\mu} \cdot e_{\nu}}{\partial_{1} W_{0}(x) \cdots \partial_{5} W_{0}(x)}, \\
C_{\mu \nu \lambda}=C_{\mu \nu}^{\sigma} \eta_{\sigma \lambda} & =\operatorname{Res} \frac{e_{\mu} \cdot e_{\nu} \cdot e_{\lambda}}{\partial_{1} W_{0}(x) \cdots \partial_{5} W_{0}(x)} .
\end{aligned}
$$

We consider a general non-necessarily homogeneous deformation

$$
W(x)=W_{0}(x)+\sum t^{\mu} e_{\mu}(x)
$$

The space of parameters $t^{\mu}$ then possesses a structure of a Frobenius manifold $\mathcal{M}_{F}$. That is, there are a multiplication and a flat metric $h_{\mu \nu}(t)$ on the monodromy invariant Milnor

\footnotetext{
${ }^{3}$ For the quintic threefold $W_{0}(x)=\sum_{i} x_{i}^{5}, \operatorname{dim} R_{0}=1024, \operatorname{dim} R_{0}^{Q}=204$, there are 101 elements of $R_{0}^{Q}$ of the weight $d$. Q in the notation $R_{0}^{Q}$ stands for the so-called quantum symmetry group (see, e.g., section 6 ). Here and after one can consider the same construction with a bigger quantum symmetries group $\mathbb{Z}_{d} \subset Q$.
} 
$\operatorname{ring} R^{Q}$ of the deformed singularity $W(x)$ :

$$
R=\frac{\mathbb{C}\left[x_{1}, \ldots, x_{5}\right]}{\partial_{1} W(x) \cdot \ldots \cdot \partial_{5} W(x)}, R^{Q}=\{e \in R, \mid[e] \in d \cdot \mathbb{Z}\}
$$

Since the metric $h_{\mu \nu}$ is flat, there exist flat coorinates $s^{\mu}$ on $\mathcal{M}_{F}$. The metric in these coordinates is the constant $\eta_{\mu \nu}$ equal to $h_{\mu \nu}(t=0)$. The structure constants of the ring $R$ are given by the third derivatives of a function $F(t)$ called the Frobenius potential,

$$
C_{\mu \nu}^{\rho}(t) h_{\rho \sigma}=\nabla_{\mu} \nabla_{\nu} \nabla_{\sigma} F(t)
$$

where $\nabla$ is Levi-Civita connection for $h_{\mu \nu}(t)$.

We recall Riemannian manifold $\mathcal{M}_{F}$ with a flat metric $h_{\mu \nu}(t)$ is called a Frobenius manifold if each tangent space of $\mathcal{M}_{F}$ is a Frobenius algebra with a scalar product given by $h_{\mu \nu}$ and the structure constants satisfy integrability condition which implies (4.7) for some local function $F(t)$ (see [7] for more details on FM).

\section{Frobenius manifold and Cohomology group $\mathbf{H}_{\mathbf{D}^{ \pm}}^{5}\left(\mathbb{C}^{5}\right)$}

We consider the differentials

$$
D^{ \pm}=D_{W_{0}}^{ \pm}=\mathrm{d} \pm \mathrm{d} W_{0} \wedge
$$

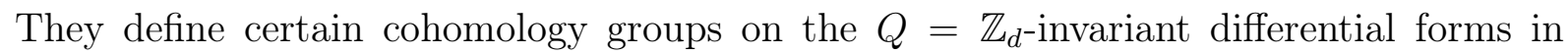
$\mathbb{C}^{5}$. The fifth cohomology groups $H_{D^{ \pm}}^{5}\left(\mathbb{C}^{5}\right)_{w \in d \cdot \mathbb{Z}}$ of this differentials as linear spaces are isomorphic to the invariant Milnor ring $R^{Q}$ [12]. This isomorphism is given by $e_{\mu}(x) \rightarrow$ $e_{\mu}(x) \mathrm{d}^{5} x[22,23]$.

The cohomology group $H_{D^{\mp}}^{5}\left(\mathbb{C}^{5}\right)_{w \in d \cdot \mathbb{Z}}$ is dual to the homology group $H_{5}\left(\mathbb{C}^{5}, \operatorname{Re} W_{0}(x)=\mp \infty\right)_{w \in d \cdot \mathbb{Z}}$ which is by definition a $Q=\mathbb{Z}_{d}$ invariant subgroup of $H_{5}\left(\mathbb{C}^{5}, \operatorname{Re} W_{0}(x)=\mp \infty\right)$. This duality follows from the self-consistency of the perfect pairing between these groups defined as

$$
\left\langle\Gamma_{\mu}^{ \pm}, e_{\nu} \mathrm{d}^{5} x\right\rangle=\int_{\Gamma_{\mu}^{ \pm}} e_{\nu} \cdot e^{\mp W_{0}(x)} \mathrm{d}^{5} x
$$

As was already mentioned, $H_{5}\left(\mathbb{C}^{5}, \operatorname{Re} W_{0}(x)=\mp \infty\right)_{w \in d \cdot \mathbb{Z}}$ is isomorphic to $H^{3}(X)$. Therefore we have an isomorphism $R^{Q} \simeq H^{3}(X)$, moreover, under this isomorphism weight decomposition on the one side becomes Hodge decompositon on the other. Namely there is a natural way to construct a corresponding harmonic form from the polynomial in $R^{Q}$ [11].

Furthermore we define a set of cycles $\Gamma_{\mu}^{ \pm}$in the group $H_{5}\left(\mathbb{C}^{5}, \operatorname{Re} W_{0}(x)= \pm \infty\right)_{w \in d \cdot \mathbb{Z}}$ by requiring that

$$
\int_{\Gamma_{\mu}^{ \pm}} e_{\nu} \cdot e^{\mp W_{0}(x)} \mathrm{d}^{5} x=\delta_{\nu}^{\mu}
$$

with the Kroneker matrix $\delta_{\nu}^{\mu}$.

The convenient computation technique in $H_{D^{ \pm}}\left(\mathbb{C}^{5}\right)$ introduced in $[13,23]$ can be used 
to compute the integrals

$$
\int_{\Gamma_{\mu}^{ \pm}} e_{\nu} \cdot e^{\mp W(x, \phi)} \mathrm{d}^{5} x
$$

$W(x, \phi)=W_{0}(x)+\sum_{s=0}^{\mu} \phi^{s} e_{s}(x)$. Let us review it in application to our case.

First we expand the exponent in the integral above in $\phi$ :

$$
\sigma_{\mu}^{ \pm}(\phi)=\sum_{m}\left(\prod_{s} \frac{\left( \pm \phi_{s}\right)^{m_{s}}}{m_{s} !}\right) \int_{\Gamma_{\mu}^{ \pm}} \prod_{s} e_{s}(x)^{m_{s}} e^{\mp W_{0}(x)} \mathrm{d}^{5} x .
$$

We note, that $\sigma_{\mu}^{-}(\phi)=(-1)^{|\mu|} \sigma_{\mu}^{+}(\phi)$, so we focus on $\sigma_{\mu}(\phi):=\sigma_{\mu}^{+}(\phi)$.

For each of the summands in (4.12) form $\prod_{s} e_{s}(x)^{m_{s}} \mathrm{~d}^{5} x$ belongs to $H_{D_{ \pm}}^{5}\left(\mathbb{C}^{5}\right)_{i n v}$, because it is $Q$-invariant. Thus, we can expand the form in the basis $e_{\mu}(x) \mathrm{d}^{5} x \in$ $H_{D_{ \pm}}^{5}\left(\mathbb{C}^{5}\right)_{i n v}$. Namely we always can find such a polynomial 4 -form $U$, that

$$
\prod_{s} e_{s}(x)^{m_{s}} \mathrm{~d}^{5} x=\sum_{\nu} C_{\nu}(m) e_{\nu}(x) \mathrm{d}^{5} x+D_{+} U
$$

Therefore, the integral in (4.12) is reduced to

$$
\int_{\Gamma_{\mu}^{ \pm}} \prod_{s} e_{s}(x)^{m_{s}} e^{\mp W_{0}(x)} \mathrm{d}^{5} x=C_{\mu}(m) .
$$

The computation of $C_{\mu}(m)$ and $U$ can be done as follows. Let degree of $\prod_{s} e_{s}(x)^{m_{s}} \mathrm{~d}^{5} x$ be $k d$. Since each of differentials $D_{ \pm}$is the sum of two quasihomogeneous terms of degree 0 and degree $d$, it is convenient to present the $4-$ form $U$ as a sum $U=\sum_{j=1}^{k-1} U_{j}$ with $\mathrm{d} U_{j}$ quasihomogeneous of degree $j d$ and $\mathrm{d} W_{0} \wedge U_{j}$ of degree $(j+1) d$.

Substituting to (4.13) arrive to the following system of equations:

$$
\begin{aligned}
& \prod_{s} e_{s}(x)^{m_{s}} \mathrm{~d}^{5} x=\mathrm{d} W_{0} \wedge U_{k-1}, \\
& \mathrm{~d} U_{j}=\mathrm{d} W_{0} \wedge U_{j-1}, 4<j<k-1, \\
& \mathrm{~d} U_{j}=\mathrm{d} W_{0} \wedge U_{j-1}+\sum_{\nu, \operatorname{deg}(\nu)=j d} C_{\nu}(m) e_{\nu}(x) \mathrm{d}^{5} x, 1 \leq j \leq 4,
\end{aligned}
$$

where $U_{0}:=0$.

The system above can be tranformed to a recursion for the coefficients $C_{\mu}(m)$ in $m$. To get the recursion let us write $\mathrm{d} U_{k-1}=\sum_{m^{\prime}} c\left(m, m^{\prime}\right) \prod_{s} e_{s}(x)^{m_{s}^{\prime}} \mathrm{d}^{5} x$ for some constants $c\left(m, m^{\prime}\right)$. Then we can find these constants from the first equation of (4.15). This gives the relation $C_{\mu}(m)=\sum_{m^{\prime}} c\left(m, m^{\prime}\right) C_{\mu}\left(m^{\prime}\right)$, where $\operatorname{deg} \prod_{s} e_{s}(x)^{m_{s}^{\prime}}<\operatorname{deg} \prod_{s} e_{s}(x)^{m_{s}}$. We will demonstrate it explicitly below for the quintic example.

\section{Moduli space as a subspace of the Frobenius manifold}

In general, $W(x)=W_{0}(x)+\sum t^{\mu} e_{\mu}(x)=0$ does not define a surface in a projective space. This only occurs when $W(x)$ is quasihomogeneous, i.e. in a case of marginal 
deformations or deformations that have the same scaling property as $W_{0}(x)$. We let denote $\left\{\phi^{s}\right\} \subset\left\{t^{\alpha}\right\}$ the marginal deformation parameters.

Thus, the marginal deformations $W_{0}(x)+\sum \phi^{s} e_{s}(x)$ define a subspace of a total Frobenius manifold connected with the LG superpotential $W_{0}$. This marginal subspace of the Frobenius manifold coincides (at least locally and maybe after some orbifolding by a finite group) with the moduli space of the CY manifold.

\section{Computing the Kähler potential.}

\section{The second basis of periods.}

In this section we use the connection of the CY moduli space to the corresponding FM to find the inverse intersection matrix of the cycles $C^{\mu \nu}, q_{\mu} \cap q_{\nu}=Q_{\mu}^{+} \cap Q_{\nu}^{-}$.

For this, we define a few additional periods $\omega_{\alpha, \mu}^{ \pm}(\phi)$ as integrals of $e_{\alpha}(x) \mathrm{d}^{5} x$ from $H_{D^{ \pm}}^{5}\left(\mathbb{C}^{5}\right)$ over the cycles $Q_{\mu}^{ \pm} \in H_{5}\left(\mathbb{C}^{5}, \operatorname{Re} W_{0}(x)= \pm \infty\right)_{w \in d \cdot \mathbb{Z}}$ that have been defined earlier:

$$
\omega_{\alpha \mu}^{ \pm}(\phi)=\int_{Q_{\mu}^{ \pm}} e_{\alpha}(x) e^{\mp W(x, \phi)} \mathrm{d}^{5} x
$$

In particular, the periods $\omega_{1 \mu}^{ \pm}(\phi)$ coincide with the periods $\omega_{\mu}^{ \pm}(\phi)$ defined above since we assume that $e_{1}(x)=1$ denotes the unity in the ring $R$.

The crucial fact for the possibility to compute the intersection matrix $C^{\mu \nu}$ is its connection (as proved in the appendix) with the Frobenius metric $h_{\alpha \beta}(t)$ for $t=0[15,26]$ as:

$$
\eta_{\alpha \beta}=\omega_{\alpha, \mu}^{+}(t=0) C^{\mu \nu} \omega_{\beta, \nu}^{-}(t=0) .
$$

Formula (5.2) in principle allows us to determine the inverse intersection matrix $C^{\mu \nu}$. It turns out to be convenient to use the following maneuver.

We define another basis of periods $\sigma_{\mu}^{ \pm}(\psi)$ as integrals over the cycles $\Gamma_{\mu}^{ \pm} \in$ $H_{5}\left(\mathbb{C}^{5}, \operatorname{Re} W_{0}(x)= \pm \infty\right)_{w \in d \cdot \mathbb{Z}}$ :

$$
\sigma_{\mu}^{ \pm}(\psi)=\int_{\Gamma_{\mu}^{ \pm}} e^{\mp W(x, \psi)} \mathrm{d}^{5} x,
$$

where $\Gamma_{\mu}^{ \pm}$is chosen dual to $e_{\mu}(x) \mathrm{d}^{5} x$ as explained above.

Once we have an oscillatory representation for the periods $\sigma_{\mu}^{ \pm}(\phi)$ over the corresponding cycles $\Gamma_{\mu}^{ \pm}$, we can define additional integrals $\sigma_{\alpha, \mu}^{ \pm}(\phi)$ over the same cycles as

$$
\sigma_{\alpha, \mu}^{ \pm}(\phi)=\int_{\Gamma_{\mu}^{ \pm}} e_{\alpha}(x) e^{\mp W(x, \phi)} \mathrm{d}^{5} x .
$$

It follows from $e_{1}(x)=1$ that $\sigma_{1 \mu}^{ \pm}=\sigma_{\mu}^{ \pm}$. Due to our choice of the cycles $\Gamma_{\mu}^{ \pm}$we also have $\sigma_{\alpha, \mu}^{ \pm}(t=0)=\delta_{\alpha, \mu}$. 


\section{The construction.}

Since both $\omega_{\alpha, \mu}^{ \pm}(\phi)$ and $\sigma_{\alpha, \nu}^{ \pm}(\phi)$ are basises of periods defined as the integrals over the cycles in the same space $H_{5}\left(\mathbb{C}^{5}, \operatorname{Re} W_{0}(x)= \pm \infty\right)$, they are connected by some constant matrix $\left(T^{ \pm}\right)_{\mu}^{\nu}$, which is independent of $\alpha$ :

$$
\omega_{\alpha, \mu}^{ \pm}(\phi)=\left(T^{ \pm}\right)_{\mu}^{\nu} \sigma_{\alpha, \nu}^{ \pm}(\phi)
$$

Therefore, to find the matrix $T$, it suffices to take a few first terms of the expansion over $\phi$ of the periods $\omega_{\mu}^{ \pm}(\phi)$ and $\sigma_{\mu}^{ \pm}(\phi)$ and to use the equation

$$
\omega_{\mu}^{ \pm}(\phi)=\left(T^{ \pm}\right)_{\mu}^{\nu} \sigma_{\nu}^{ \pm}(\phi)
$$

Also, knowing that $\sigma_{\alpha, \mu}^{ \pm}(\phi=0)=\delta_{\alpha, \mu}$, we get

$$
\omega_{\alpha, \mu}^{ \pm}(\phi=0)=\left(T^{ \pm}\right)_{\mu}^{\alpha}
$$

From eq. (5.2) we then obtain

$$
\eta^{\mu \nu}=\left(T^{+}\right)_{\rho}^{\mu} C^{\rho \sigma}\left(T^{-}\right)_{\sigma}^{\nu}
$$

From this we find the intersection matrix $C^{\rho \sigma}$ in terms of the known Frobenius metric $\eta^{\mu \nu}$ and the also known matrix $T$.

From (3.14), (5.6), and (5.8), we finally conclude that

$$
e^{-K(\phi)}=\sigma_{\mu}(\phi) \eta^{\mu \nu} M_{\nu}^{\lambda} \overline{\sigma_{\lambda}^{-}(\phi)}
$$

where the matrix $M_{b}^{a}=\left(T^{-1}\right)_{c}^{a} \bar{T}_{b}^{c}$.

This formula is our main result. It gives an explicit expression for the Kähler potential $K$ in terms of the periods $\sigma_{\mu}(\psi)$,

FM metric $\eta_{\mu \nu}$ and matrix $M_{\nu}^{\mu}$. All these data can be computed exactly as it has been explained above.

It makes sense to stress that having the exact expression for $\omega_{\nu}^{ \pm}(\phi)$, we obtain the exact and explicit expressions for the periods $\sigma_{\mu}^{ \pm}(\phi)$ from (5.6):

$$
\sigma_{\mu}^{ \pm}(\phi)=\left(\left(T^{ \pm}\right)^{-1}\right)_{\mu}^{\nu} \omega_{\nu}^{ \pm}(\phi)
$$

In terms of the periods $\sigma_{\mu}^{ \pm}(\phi)$ expression (5.9) for the Kähler potential has a more convenient form for calculating the metric on the CY moduli space.

\section{$6 \quad$ Examples}

\section{Quintic CY threefold}

This CY manifold is defined as

$$
X_{\psi}=\left\{x_{i} \in \mathbb{P}^{4} \mid W_{\psi}(x)=x_{1}^{5}+x_{2}^{5}+x_{3}^{5}+x_{4}^{5}+x_{5}^{5}-5 \psi x_{1} x_{2} x_{3} x_{4} x_{5}=0\right\} .
$$


Here we set $\phi_{0}=5 \psi$ to connect with [1].

We compute the complex structure moduli space metric for this one-parameter family. In this case, the phase symmetry(see section 3 ) is $\mathbb{Z}_{5}^{5}$ and the induced action $\mathcal{A}$ on the one-dimensional space $\{\psi\}$ is $\mathbb{Z}_{5}: \psi \rightarrow e^{2 \pi i / 5} \psi$.

This space is the whole complex structure moduli space of the quotient $X / \mathbb{Z}_{5}^{3}=: \hat{X}$, that is the mirror manifold of the original quintic. In particular, $h_{1,1}(\hat{X})=101, h_{2,1}(\hat{X})=1$. We choose cycles $\Gamma_{\mu}^{ \pm}$dual to the cohomology classes $\mathrm{d}^{5} x, \prod x_{i} \cdot \mathrm{d}^{5} x, \prod x_{i}^{2} \cdot \mathrm{d}^{5} x, \prod x_{i}^{3} \cdot \mathrm{d}^{5} x$, that form a basis in the cohomology subgroup corresponding to the subring of Milnor ring elements of weight $\in 5 \mathbb{Z}$ and invariant under the $\mathbb{Z}_{5}^{3}$ action of the phase symmetry. For the periods, the algorithm, described above gives:

$$
\sigma_{\mu}^{-}(\psi)=\sum_{m}\left(\frac{(-5 \psi)^{m}}{m !}\right) \int_{\Gamma_{\mu}^{-}}\left(x_{1} x_{2} x_{3} x_{4} x_{5}\right)^{m} e^{\sum_{i} x_{i}^{5}} \mathrm{~d}^{5} x .
$$

Let $m=5 n+\nu, \nu<5$. We want to expand

$$
\prod x_{i}^{5 n+\nu} \mathrm{d}^{5} x=\sum_{\nu} C_{\mu}(m)\left(x_{1} x_{2} x_{3} x_{4} x_{5}\right)^{\mu} \mathrm{d}^{5} x+D_{+} U
$$

Note that

$$
\begin{aligned}
D_{+}\left(\frac { 1 } { 5 } x _ { 1 } ^ { 5 n + k - 4 } f \left(x_{2}, \cdots,\right.\right. & \left.\left.x_{5}\right) \mathrm{~d} x_{2} \wedge \cdots \wedge \mathrm{d} x_{5}\right)= \\
= & {\left[x_{1}^{5 n+k}+\left(n+\frac{k-4}{5}\right) x_{1}^{5(n-1)+k}\right] f\left(x_{2}, \cdots, x_{5}\right) \mathrm{d}^{5} x }
\end{aligned}
$$

where $f$ denotes an arbitrary polynomial. Applying it to $f=\left(x_{2} x_{3} x_{4} x_{5}\right)^{5 n+k}$ we get

$$
\prod x_{i}^{5 n+\nu} \mathrm{d}^{5} x=-\left(n+\frac{k-4}{5}\right) x_{1}^{5(n-1)+\nu}\left(x_{2} x_{3} x_{4} x_{5}\right)^{5 n+\nu} \mathrm{d}^{5} x \text { modulo } D_{+}(\cdots)
$$

We use an analogous formula for permuted $x_{1}, \ldots, x_{5}$ and appropriate $f$ to get the following recursion:

$$
\prod x_{i}^{5 n+\nu} \mathrm{d}^{5} x=-\left(n+\frac{k-4}{5}\right)^{5} \prod x_{i}^{5(n-1)+\nu} \mathrm{d}^{5} x+D_{+} \tilde{U}
$$

which implies

$$
C_{\mu}(m)=-\left(n+\frac{k-4}{5}\right)^{5} C_{\mu}(m-5) .
$$

This recursion is immediately solved

$$
\begin{gathered}
\prod x_{i}^{5 n+\nu} \mathrm{d}^{5} x=(-1)^{n} \frac{\Gamma(n+\nu / 5)^{5}}{\Gamma(\nu / 5)} \prod x_{i}^{\nu} \mathrm{d}^{5} x+D_{+} U \\
C_{\mu}(m)=\delta_{\mu, \nu}(-1)^{n} \frac{\Gamma(n+\nu / 5)^{5}}{\Gamma(\nu / 5)}
\end{gathered}
$$


Plugging it into (6.2) we obtain

$$
\sigma_{\mu}^{ \pm}(\psi)=\frac{( \pm 1)^{\mu-1}}{\Gamma(\mu / 5)^{5} 5^{\mu} \psi} \sum_{n=0}^{\infty} \frac{\Gamma^{5}(n+\mu / 5)}{\Gamma(5 n+\mu)}(5 \psi)^{5 n+\mu}
$$

which have the following behaviour $( \pm \psi)^{\mu-1} / \Gamma(\mu)+O\left(\psi^{\mu+4}\right)$.

The fundamental period for the quintic is defined as a residue of a holomorphic threeform

$$
\frac{x_{5} \mathrm{~d} x_{1} \wedge \mathrm{d} x_{2} \wedge \mathrm{d} x_{3}}{\partial P_{\psi} / \partial x_{4}},
$$

that is given by an integral over a cycle $q_{1}$, which is three-dimensional torus [1]. Its analytic continuations as explained above give the whole basis of periods in a basis of cycles with integral coefficients:

$$
\omega_{\mu}(\psi)=\sum_{m=1}^{\infty} \frac{e^{4 \pi i m / 5} \Gamma(m / 5)\left(5 e^{2 \pi i(\mu-1) / 5} \psi\right)^{m-1}}{\Gamma(m) \Gamma^{4}(1-m / 5)}, \quad|\psi|<1,
$$

in this case $\omega_{\alpha, \mu}(0)$ equals to $\partial_{\psi}^{\alpha-1} \omega_{\mu}(\psi)$ for $\alpha=1, . ., 4$.

Therefore, taking the first four terms of the expansion of the periods above and using (5.7) we obtain

$$
T_{\nu}^{\mu}=\frac{e^{2 \pi i(\nu-1)(\mu-1) / 5} e^{4 \pi i \nu / 5} 5^{\nu-1} \Gamma(\nu / 5)}{\Gamma^{4}(1-\nu / 5)} .
$$

The FM holomorphic metric in this case

$$
\eta=\operatorname{antidiag}(1,1,1,1)
$$

Finally, we obtain $\eta M=\eta T^{-1} \bar{T}$ and the Kähler potential for the metric according to our main formula (5.9):

$$
e^{-K(\psi)}=\frac{\Gamma^{5}(1 / 5)}{125 \Gamma^{5}(4 / 5)} \sigma_{11}^{+} \overline{\sigma_{11}^{-}}+\frac{\Gamma^{5}(2 / 5)}{5 \Gamma^{5}(3 / 5)} \sigma_{12}^{+} \overline{\sigma_{12}^{-}}+\frac{5 \Gamma^{5}(3 / 5)}{\Gamma^{5}(2 / 5)} \sigma_{13}^{+} \overline{\sigma_{13}^{-}}+\frac{125 \Gamma^{5}(4 / 5)}{\Gamma^{5}(1 / 5)} \sigma_{14}^{+} \overline{\sigma_{14}^{-}} .
$$

In particular,

$$
G_{\psi \bar{\psi}}(0)=25 \frac{\Gamma^{5}(4 / 5) \Gamma^{5}(2 / 5)}{\Gamma^{5}(1 / 5) \Gamma^{5}(3 / 5)}
$$

\section{Fermat hypersurfaces}

The direct generalization of the quintic is a Fermat hypersurface, which is the one given by the equation

$$
W_{0}(x)=\sum_{i=1}^{5} x_{i}^{n_{i}}, \quad n_{i}=d / k_{i}, \quad \sum k_{i}=d,
$$

and the degree $d$ is equal to the least common multiple of $\left\{k_{i}\right\}$.

As in the case above, we consider a one-dimensional deformation $W\left(x, \phi_{0}\right)=W_{0}(x)+$ 
$\phi_{0} \prod_{i=1}^{5} x_{i}$. The phase symmetry group is $\Pi_{X}=\mathbb{Z}_{n_{1}} \times \cdots \times \mathbb{Z}_{n_{5}}$. The lifted action on $\phi_{0}$ is $\mathbb{Z}_{d}: \phi_{0} \rightarrow \zeta \phi_{0}, \zeta=e^{2 \pi i / d}$. We take the expression for the fundamental period from [2]:

$$
\omega_{1}\left(\phi_{0}\right)=\sum_{r=1}^{d-1} A(r) \frac{\phi_{0}^{r-1}}{\Gamma(r)}+O\left(\phi_{0}^{d-1}\right)
$$

and

$$
A(r)=\frac{(-1)^{r-1} e^{\frac{-\pi i r}{d}}}{\sin \frac{r \pi}{d} \prod_{i=1}^{5} \Gamma\left(1-\frac{k_{j} r}{d}\right)} .
$$

We note that $A(r)$ vanishes if $k_{i} r / d \in \mathbb{Z}$, i.e. $r / n_{i} \in \mathbb{Z}$. According to the general analytic continuation procedure

$$
\omega_{\mu}\left(\phi_{0}\right)=\sum \zeta^{(r-1)(\mu-1)} A(r) \frac{\phi_{0}^{r-1}}{\Gamma(r)}+O\left(\phi_{0}^{d-1}\right)
$$

Using the definitions (4.10) and (5.3) we obtain

$$
\sigma_{\mu}^{+}\left(\phi_{0}\right)=\frac{\phi_{0}^{\mu-1}}{\Gamma(\mu)}+O\left(\phi_{0}^{\mu+d-2}\right), \quad \mu / n_{i} \notin \mathbb{Z}, \text { otherwise } 0
$$

This latter condition implies that $\omega_{\mu}$ form a basis in the periods of $\Omega$ deformed by $\phi_{0}$. We obtain the transition matrix

$$
\begin{aligned}
& T_{\nu}^{\mu}=\zeta^{(\mu-1)(\nu-1)} A(\mu), \quad \mu / n_{i} \notin \mathbb{Z}, \quad \nu / n_{i} \notin \mathbb{Z} \\
& \left(T^{-1}\right)_{\mu}^{\lambda}=\frac{\bar{\zeta}^{(\lambda-1)(\nu-1)}}{\tilde{d}-1} \frac{1}{A(\mu)}
\end{aligned}
$$

and the real structure

$$
M_{\nu}^{\mu}=\frac{\bar{A}(\mu)}{A(d-\mu)} \delta_{\mu+\nu, d} .
$$

In this case, $\eta_{\mu, \nu}=\delta_{\mu+\nu, d}$ and therefore

$$
e^{-K\left(\phi_{0}\right)}=\sum_{\mu=1, \mu / n_{i} \notin \mathbb{Z}}^{d-1} \prod_{i=1}^{5} \gamma\left(\frac{k_{i} \mu}{d}\right) \sigma_{\mu}^{+}\left(\phi_{0}\right) \overline{\sigma_{\mu}^{-}\left(\phi_{0}\right)}
$$

where $\gamma(x)=\Gamma(x) / \Gamma(1-x)$ and

$$
\sigma_{\mu}^{ \pm}\left(\phi_{0}\right)= \pm \sum_{R=0}^{\infty} \frac{\phi_{0}^{\mu-1+d R}}{\Gamma(d R+\mu)} \prod_{j=1}^{5} \frac{\Gamma\left(k_{j}\left(R+\frac{\mu}{d}\right)\right)}{\Gamma\left(\frac{k_{j} \mu}{d}\right)} .
$$

From this we get a formula for the metric itself

$$
G_{\phi_{0} \overline{\phi_{0}}}=\prod_{i=1}^{5}\left(\gamma\left(\frac{k_{i} \mu_{0}}{d}\right) \gamma\left(1-\frac{k_{i}}{d}\right)\right) \frac{\left|\phi_{0}\right|^{2\left(\mu_{0}-1\right)}}{\Gamma\left(\mu_{0}\right)^{2}}+O\left(\left|\phi_{0}\right|^{2 \mu_{0}}\right),
$$

where $\mu_{0}$ is the least integer $1 \leq \mu_{0}<d$ such that $\left(\mu_{0}+1\right) / n_{j} \neq \mathbb{Z}$. The last formula 
reproduces the known results for CY manifolds $\mathbb{P}_{(2,1,1,1,1)}^{4}[6], \mathbb{P}_{(4,1,1,1,1)}^{4}[8]$ and $\mathbb{P}_{(5,2,1,1,1)}^{4}[10]$ obtained in [16].

\section{The case of invertible polynomial}

We assume that the above approach is applicable to the case of CY manifold defined in terms of the hypersurface in weighted projective spaces of the type described in [2], i.e., the hypersurfaces whose defining polynomial is

$$
W_{0}(x)=\sum_{j=1}^{5} \prod_{i=1}^{5} x_{i}^{a_{i j}}, \quad \sum k_{i} a_{i j}=d,
$$

and

$$
\sum k_{i}=d
$$

In this case periods are given in terms of the mirror CY manifold $\hat{X}$. We briefly describe the construction of $\hat{X}$ following [6].

The polynimial $W_{0}(x)$ has a group $\Pi_{X}$ of phase symmetries represented as

$$
\Pi_{X}=Q_{X} \times G_{X}
$$

where $Q_{X}$ is a quantum symmetry group $\simeq\left(\mathbb{Z}_{d}: k_{1}, \cdots, k_{5}\right)^{4}$. The complement to $Q_{X}$ in $\Pi_{X}$ is called a geometric symmetry group $G_{X}$. For mirror manifolds the total phase symmetry is unchanged whereas roles of quantum and geometric symmetries switch:

$$
G_{X}=Q_{\hat{X}}, \quad Q_{X}=G_{\hat{X}}
$$

To build such a mirror, we must first to consider a polynomial $\hat{W}_{0}(x)$ with a transposed matrix of exponents $\hat{a}_{i j}=a_{j i}$,

$$
\hat{W}_{0}(x)=\sum_{j=1}^{5} \prod_{i=1}^{5} x_{i}^{\hat{a}_{i j}}, \quad \sum \hat{k}_{i} a_{j i}=\hat{d}
$$

and

$$
\sum \hat{k}_{i}=\hat{d}
$$

Here $\hat{k}_{i}$ and $\hat{d}$ are uniquely defined by the reqirement that the equalities above are satisfied.

This polynomial has the same group of phase symmetries, however generically the condition (6.27) is not fulfiled, i.e. its quantum symmetry is smaller, then geometric symmetry of the original hypersurface.

To get a mirror we need to enlarge quantum symmetry of $\left\{\hat{W}_{0}(x)=0\right\}$. For this purpose we take a quotient of the hypersurface $\left\{\hat{W}_{0}(x)=0\right\} / H$, where $H$ is some subgroup of phase symmetries which is to be found in each case [6].

\footnotetext{
${ }^{4}$ Notations are the same as in [6], that is $1 \in\left(\mathbb{Z}^{r}: a_{1}, \cdots, a_{5}\right)$ acts as $x_{i} \rightarrow e^{2 \pi i a_{i} / r} x_{i}$. We note that action of the quantum symmetries on $X$ is trivial.
} 
Thus, computing complex moduli space for the manifold $X$ (or $\hat{X}$ ) we compute also a complexified Kähler moduli space metric for the mirror CY through the mirror map.

The periods $\omega_{\mu}(\phi)$ in this case were computed in (6.25) in [2] and, if we set all parameters $\phi^{s}$ (but not $\phi_{0}$ ) equal to zero for simplicity, then we have:

$$
\begin{array}{r}
\omega_{1}\left(\phi_{0}\right)=\sum_{r=1}^{\hat{d}-1} A(r) \frac{\phi_{0}^{r-1}}{\Gamma(r)}+O\left(\phi_{0}^{\hat{d}-1}\right) \\
A(\mu)=(-1)^{\mu} \frac{\pi}{\hat{d} \sin \frac{\pi \mu}{\hat{d}}} \prod_{j=1}^{5} \frac{1}{\Gamma\left(1-\frac{\hat{k}_{j} \mu}{\hat{d}}\right)} .
\end{array}
$$

For our general method to work, this must give all relevant periods. Basically we must check that all possible periods are obtained from this one (with all $\phi^{s} \neq 0$ ) by phasesymmetry analytic continuations. In other words it is necessary to verify the relation

$$
\operatorname{dim}\left\langle\omega_{0}(\mathcal{A}(g) \cdot \phi)\right\rangle_{g \in G_{X}}=\operatorname{dim} H_{3}(X)
$$

This was certainly the case in the preceding examples, but we are not aware of this fact in general (it is so in all examples). As in the previous example, in the one-modulus case we obtain

$$
e^{-K\left(\phi_{0}\right)}=\sum_{\mu=1, \mu \hat{k}_{i} / \hat{d} \notin \mathbb{Z}}^{\hat{d}-1} \eta^{\mu, \hat{d}-\nu} \prod_{j=1}^{5} \gamma\left(\frac{\hat{k}_{j} \mu}{\hat{d}}\right) \sigma_{\mu}^{+}\left(\phi_{0}\right) \overline{\sigma_{\nu}^{-}\left(\phi_{0}\right)}
$$

For this formula to hold the number of linearly independent elements $\prod_{i=1}^{5} x_{i}^{n} \mathrm{~d}^{5} x \in$ $H_{D^{ \pm}}^{5}\left(\mathbb{C}^{5}\right)$ should be equal to the number of $1 \leq \mu<\hat{d}, \mu k_{i} / d \neq \mathbb{Z}$.

If $\hat{H} \subset G_{X}$, we may also restrict all the computations to the case of $\hat{H}$-invariant deformations and homology elements respectively (to describe a mirror manifold, for instance).

\section{Conclusion}

A new method for computing the CY moduli space metric is proposed. It can be used for a larger class of of CY manifolds than this have been done before. This method does not demand using of Picard-Fuchs equations. Instead of this, the cohomology technique used in [23] for computing of the FM flat coordinates [24, 25] can be applied for computing the periods. Therefore, as we expect, it can be applied for the computations of the CY moduli space geometry in cases when the dimesion of the moduli space is more than one. The FM structure naturally arising from an $\mathrm{N}=2$ superconformal theory plays a significant role in our computation. The result is given in terms of the topological metric on the latter manifold and two basises of periods, both of which we are able to compute avoiding the complicated direct computation of the symplectic basis of periods.

Our methods have been applied in this paper to CY manifolds, given by one polynomial equation, in particular to the case of Fermat hypersurfaces and to the famous quintic threefold. We suppose that the same approach can be applied to CY manifolds of a more 
general type. We also work on the problem of computing the moduli space metric around other interesting points in the moduli spaces, such as the large complex structure limit.

\section{Acknowledgements}

We are grateful to M. Bershtein, B. Everett, D. Gepner, A. Givental, A. Okounkov, A. Rosly, A. Varchenko and V. Vasiliev for the useful discussions. We are also thankful to T. Hübsch for his useful comments during the preparation of the paper. The work of A. B. on the main results presented in sections $1-5$ was performed at IITP with the financial support of the Russian Science Foundation (Grant No.14-60-00150). The work of K.A. was supported by the Foundation for the advancement of theoretical physics "BASIS".

\section{APPENDIX}

The proof of the relation (5.2)

Following [26] here we want to prove the first equality in (5.2), that is

$$
h_{a b}=\operatorname{Res} \frac{e_{a} \cdot e_{b} \mathrm{~d}^{n} x}{\partial_{1} W_{0} \cdots \partial_{n} W_{0}}=\int_{Q_{\mu}^{+}} e_{a} e^{-W_{0}} \mathrm{~d}^{n} x C^{\mu \nu} \int_{Q_{\nu}^{-}} e_{b} e^{W_{0}} \mathrm{~d}^{n} x .
$$

To do this consider a small Morse function perturbation $W(x, t)=W_{0}(x)+e_{a} t_{a}$ so that 0 - critical point of $W$ becomes a set of Morse points $p_{1}, \ldots, p_{\mu}$ and consider a bilinear form

$$
h_{a b}(t, z)=\int_{Q_{\mu}^{+}} e_{a} e^{-W(x, t) / z} \mathrm{~d}^{n} x C^{\mu \nu} \int_{Q_{\nu}^{-}} e_{b} e^{W(x, t) / z} \mathrm{~d}^{n} x .
$$

First of all we notice, that

$$
h_{a b}(t=0, z)=z^{k} \cdot h_{a b}(t=0,1)
$$

because if $t=0$, we can absorb $z$ by coordinate transform $x_{i} \rightarrow z^{k_{i} / d} x_{i}$.

Then we choose basis of cycles of so-called Lefschetz thimbles: $L_{i}^{ \pm}$starts from $p_{i}$ and goes along the gradient of $\operatorname{Re}(W(x, t))$ in positive/negative direction. With certain orientation their intersection numbers are $L_{\mu}^{+} \cap L_{\nu}^{-}=\delta_{\mu \nu}$.

Then rhs of (2) becomes in this basis:

$$
\sum_{i=1}^{\mu} \int_{L_{i}^{+}} e_{a} e^{-W(x, t) / z} \mathrm{~d}^{n} x \int_{L_{i}^{-}} e_{b} e^{W(x, t) / z} \mathrm{~d}^{n} x .
$$

Using stationary phase expansion as $z \rightarrow 0$ we obtain for a period:

$$
\int_{Q_{i}^{+}} e_{a}(x) e^{-W(x, t) / z} \mathrm{~d}^{n} x= \pm \frac{(2 \pi z)^{N / 2}}{\sqrt{\operatorname{Hess} W\left(p_{i}, t\right)}}\left(e_{a}\left(p_{i}\right)+O(z)\right) .
$$


Inserting in the formula (4) we get

$$
\begin{aligned}
& h_{a b}(t, z)= \pm \sum_{i=1}^{\mu}(2 \pi i z)^{N} \frac{e_{a}\left(p_{i}\right) \cdot e_{b}\left(p_{i}\right)}{\operatorname{Hess}\left(W\left(p_{i}, t\right)\right)}(1+O(z))= \\
&=(2 \pi i z)^{N}\left(\operatorname{Res} \frac{e_{a} \cdot e_{b} \mathrm{~d}^{n} x}{\partial_{1} W \cdots \partial_{N} W}+O(z)\right) .
\end{aligned}
$$

By analytic continuation it holds for $t=0$. Now we recall that $h_{a b}(0, z)=z^{k} \cdot h_{a b}(0,1)$. The required equality now follows from the previous formula.

\section{References}

[1] P. Candelas, X. de la Ossa,P. S. Green and L. Parkes, A Pair of Calabi-Yau manifolds as an exactly soluble superconformal field theory, Nucl.Phys. B359 (1991) 21-74

[2] P. Berglund, P. Candelas, X. de la Ossa, A. Font, T. Huebsch, D. Jancic and F. Quevedo, Periods for Calabi-Yau and Landau-Ginzburg vacua, Nucl.Phys. B355 (1991) 455

[3] P. Candelas, P. Green, T. Hübsch, Finite Distances Between Distinct Calabi-Yau Manifolds, Phys.Rev.Lett. 62 (1989) 1956-1959

[4] A. Strominger, Special geometry, Commun. Math. Phys. 133 (1990) 163

[5] P. Candelas, X. de la Ossa, Moduli Space of Calabi-Yau manifolds, Nucl.Phys. B335 (1991) 355-481

[6] P. Berglund and T. Hübsch A generalized constraction of Mirror manifolds, Nucl.Phys. B393 (1993) 377

[7] B. Dubrovin, Integrable systems in topological field theory, Nucl.Phys. B379 (1992) 627-689

[8] Claus Hertling. Frobenius Manifolds and Moduli Spaces for Singularities. Cambridge Tracts in Mathematics. Cambridge University Press, 2002.

[9] Yu I Manin. Frobenius manifolds, quantum cohomology, and moduli spaces. 2007.

[10] P. Candelas, P. S. Green, T. Hübsch, Rolling Among Calabi-Yau Vacua, Nucl.Phys. B330 (1990) 49-102

[11] P. Candelas, Yukawa couplings between (2,1)-forms , Nucl.Phys. B298 (1988) 458-492

[12] A. Arnold, A. Varchenko, S. Gusein-Zade, Singularities of Differentiable Maps, Birkhauser 1985

[13] A. Belavin, D. Gepner, Ya. Kononov, Flat coordinates for Saito Frobenius manifolds and String theory, arXiv:1510.06970

[14] V. Vasiliev's Letter to A. Belavin

[15] S. Cecotti and C. Vafa, Topological-anti-topological fusion, Nucl.Phys. B367 (1991) $359-461$

[16] A. Klemm, S. Theisen, Recent efforts in the computation of string couplings, TMF (1993) 95,2 
[17] D. Gepner, Exactly solvable string compactifications on manifolds of SU(N) Holonomy, Phys. Lett. 199 (1987) 380-388.

[18] P. Candelas, G. Horowitz, A. Strominger and E. Witten Vacuum configurations for Superstrings, Nucl.Phys. B258 (1985) 46-74

[19] W. Lerche,C. Vafa and N. Warner, Chiral rings in $N=2$ superconformal theories, Nucl.Phys. B324 (1989) 327-474

[20] E. Martinec, Algebraic geometry and effective lagrangians. Phys. lett. 217B(1989) 431.

[21] C. Vafa, N. Warner Phys.Lett. 218B (1989) 51.

[22] B. Blok, A. Varchenko, Topological conformal field theories and the flat coordinates, Internat. J. Mod. Phys.7 (1992) 1467-1490.

[23] A. Belavin, V. Belavin, Flat structures on the deformations of Gepner chiral rings, J. High Energy Phys., 10, 128 (2016)

[24] A. Belavin, B. Dubrovin, B. Mukhametzhanov, Minimal Liouville gravity correlation numbers from Douglas string equation, J. High Energy Phys., 01, 156 (2014)

[25] V. Belavin, Unitary Minimal Liouville Gravity and Frobenius manifolds, J. High Energy Phys., 07, 129 (2014)

[26] A. Chiodo, H. Iritani and Y. Ruan, Landau-Ginzburg/Calabi-Yau correspondence, global mirror symmetry and Orlov equivalence, arXiv:1201.0813 [math.AG] 